\title{
Immersive learning as an assessment and training tool for cardiopulmonary resuscitation skills
}

\section{Opinion}

Immersive simulation technology is a tool that gives an opportunity to learn, practice, repeat procedures and optimize clinical outcomes in emergency pediatrics. Immersive learning provides learners with a high-quality learning experience, excellent attention rate and $360^{\circ}$ evaluation. Simulation is a tool that helps to discover knowledge, to get skills and to assess the competencies. Learners are performing the task with the freedom to fail and face bad consequences. Simulation training is a tool for objectively assessing of resuscitation skills. Learners learn as they 'do', tracking their progress through assessments. Evaluation is the key point of medical training. No criterion standard of assessing performance currently exists. Immersive learning environment was used for the cardiopulmonary resuscition (CPR) assessment and training of practicing emergency pediatricians at the Research \& Educational Innovation Simulation Center. We developed a new scoring system for the assessment of cardiopulmonary resuscitation skills (RU patent 2523637). The benefit of the our new scoring system is an opportunity to assess each unit with different assessment ways. ${ }^{1-3}$ The purpose of this study was to determine the optimal refresher training interval for CPR skill among practicing physicians. The study was conducted in 2009-2013 and included 230 practicing emergency pediatricians. 180 participants were randomly selected in the prospective study to evaluate the cariopulmonary skills decay over time. We randomized 180 selected participants into 3 equal groups of 60 physicians by different time following first simulation assessment and training: 6-12 months, 1-2 years, 2-3 years. All 230 participants received a baseline 8 hour standard immersive training. 180 randomly selected participants received retraining at the decreted time intervals.

Standard immersive training program included lecture, skill training, clinical virtual scenarios, clinical hands-on scenarios and debriefings. We used our invented patent "Method for quality control of practical training for cardiopulmonary resuscitation in children" as a standard tool of cardiopulmonary skills assessment. In this prospective study we used six modules with real-time simulations that facilitate active learning through case management. As part of our research project we have randomized 230 participants into 2 equal groups of 115 doctors by sequence of 3 virtual and 3 hands-on clinical scenarios. For each scenario, a checklist of specific actions was developed, reflecting essential actions for a safe treatment. ${ }^{4}$ The sessions were recorded and the performances of individual participants were evaluated using a standardized scoring scheme. After finishing the course the physicians were retested and completed a numerical scale survey $(\mathrm{n}=235)$ of their perceptions about the course $(1=$ poor, $2=$ fair, $3=$ good and $4=$ excellent). Performance improved significantly after standard immersive training ( $78 \%$ vs $57 \% \mathrm{p}<0.05)$.

We have found that there was a benefit from using virual reality scenarios first in further improving resuscitation skills compared with hands-on scenarios first $(86 \%$ vs $67 \% \mathrm{p}<0.05)$. Further research revealed that there was a linear decay of cardiopulmonary skills over time: the quality of CPR has changed from $78,7 \%$ to $64,2 \%$ following 6-12 months after initial training, $43,4 \%$ and $39 \%$ following $1-2$ and 2-3 years after training respectively. According to the results achieved

\author{
Volume 8 Issue 5 - 2018 \\ I Gavryutina, B Blokhin \\ Department of outpatient and emergency pediatrics, National \\ Research Medical University, Russia
}

\begin{abstract}
Correspondence: Boris Blokhin, MD, Ph.D., Professor and Chair, Department of outpatient and emergency pediatrics, Russian National Research Medical University named after N.I. Pirogov (RNRMU), Ostrovitianov str. I, Moscow, Russia, I I7997, Email blokhinb@gmail.com
\end{abstract}

Received: September 19, 2018 | Published:September 25, 2018

using of immersive learning environment with virtual to hands-on sequence gives the maximum benefit in CPR training. ${ }^{5,6}$ The learner has to apply logical thinking to solve problems in acute situations and make the decision that sometimes can be harmful. Immersive learning gives learners a sense of reality to perform the task effectively.The recommended retraining of cardiopulmonary skills for emergency pediatricians is every year. Many studies had evaluated the knowledge and CPR performance skills of nurses, students, paramedics and found decline with time. But there are very few studies on skills retention among practicing physicians. Further investigation must include the identification of frequency of training in cardiopulmonary resuscitation for pediatricians practicing in different settings.

\section{Acknowledgements}

None.

\section{Conflict of Interest}

The author declares there is no conflict of interest.

\section{References}

1. Binkhorst M, Coopmans M, Draaisma JMT. Retention of knowledge and skills in pediatric basic life support amongst pediatricians. Eur J Pediatr. 2018;177(7):1089-1099.

2. Blokhin B, Gavryutina I, Loayza H, et al. Medical simulation in the assessment of cardiopulmonary resuscitation. Book of abstracts, 14th Congress of the International Society for Holter and Noninvasive Electrocardiology (ISHNE 2011), Moscow, 2011.

3. Kneebone R. Evaluating clinical simulations for learning procedural skills: a theory-based approach. Acad Med. 2005;80(6):549-553.

4. Niles D, Sutton RM, Donoghue A, et al. Rolling Refreshers: a novel approach to maintain CPR psychomotor skill competence. Resuscitation. 2009;80(8):909-912.

5. World Alliance for Patient Safety; WHO Patient Safety Curriculum Guide for Medical Schools. 2009.

6. Yang CW, Yen ZS, McGowan JE, et al. A systematic review of retention of adult advanced life support knowledge and skills in healthcare providers. Resuscitation. 2012;83(9):1055-1060. 